\title{
The design of the beam line of the Nagoya University Accelerator-driven Neutron Source
}

\author{
Ikuya Ito ${ }^{1}$ \\ Nagoya University \\ E-mail: ikuya@phi.phys.nagoya-u.ac.jp
}

D. Furusawa ${ }^{A}$, K. Hirota, G. Ichikawa, Y. Iwashita ${ }^{B}$, M. Kitaguchi, Y. Kiyanagi ${ }^{A}$,

Y. Menjo ${ }^{A}$, Y. Miwata ${ }^{C}$, H. M. Shimizu, K. Tsuchida ${ }^{A}$, Y. Tsuchikawa, Y. TsuritaA,

A. Uritani ${ }^{A}$, K. Watanabe ${ }^{A}$, Y. Yamagata ${ }^{C}$, A. Yamazaki ${ }^{A}$, S. Yoshihashi ${ }^{A}$

Dept.of Phys.Nagoya Univ., Guraduate School of Engineering Nagoya Univ. ${ }^{A}$,

Institute for Chemical Research Kyoto Univ. ${ }^{B}$, RIKEN ${ }^{C}$

\begin{abstract}
The Nagoya University Accelerator driven Neutron Source (NUANS) are now constructing at main campus of Nagoya University. The electrostatic accelerator is used with the maximum proton energy and power of $2.8 \mathrm{MeV}, 15 \mathrm{~mA}(42 \mathrm{~kW})$ respectively. Two neutron beam lines are planned at NUANS. The first beam line is dedicated to BNCT. The second beam line is designed for research and development of neutron devices and neutron imaging. The neutrons used for the 2 nd beam line are generated by using the $(\mathrm{p}, \mathrm{n})$ reaction of beryllium. We designed a compact target station for the $2 \mathrm{nd}$ beam line with the dimension of $90 \mathrm{~cm} \times 90 \mathrm{~cm} \times 95 \mathrm{~cm}$ by using radiation shielding simulation. We considered neutron beam extraction with the cross section of $10 \mathrm{~cm} \times 10 \mathrm{~cm}$ for radiography at the 2 nd beam line. The neutron flux is expected to be about $10^{4} \mathrm{n} \mathrm{cm}^{-2} \mathrm{~s}^{-1}$ with the energy less than $0.1 \mathrm{eV}$ at the position of $2 \mathrm{~m}$ from the neutron source. We also calculated the proton beam transport from the exit of the accelerator to the neutron production target of the 2 nd beam line.
\end{abstract}

The 3rd International Symposium on "Quest for the Origin of Particles and the Universe"

5-7 January 2017

Nagoya University, Japan

${ }^{1}$ Ikuya Ito 


\section{Introduction}

Neutron is a powerful tool to study fundamental physics, material science, industrial and medical applications. We are now developing various neutron devices, for example, high speed neutron detector to search for time - reversal violation in neutron resonance capture reactions. In order to accelerate the research and development, the frequent uses of neutron beam are desired. One of the solution to get the opportunity to use the beam is constructing compact neutron source.

Nagoya University Accelerator-driven Neutron Source (NUANS) is a compact neutron source using low energy $(p, n)$ reaction for multipurpose. We are currently designing two beam lines. The 1st beamline is aimed at developing equipment for Boron Neutron Capture Therapy (BNCT). The 2nd beam line is aimed at developing devices such as neutron detectors and neutron imaging. Fig.1 shows a schematic diagram of NUANS.

The proton beam accelerator is the dynamitronmanufactured by IBA company. It provides aDC proton beam with variable energy from $1.9 \mathrm{MeV}$ to $2.8 \mathrm{MeV}$. Proton beam current can be output up to $15 \mathrm{~mA}$.

We use $\mathrm{Li}(\mathrm{p}, \mathrm{n})$ reaction and $\mathrm{Be}(\mathrm{p}, \mathrm{n})$ reaction to generate neutrons. Fig.2 shows the relationship between the energy of the proton beam and the neutron yield of each nuclear reaction. The neutron yield with lithium target is one order larger than that of beryllium at the proton beam energy of $2.8 \mathrm{MeV}$. Because the neutron intansity is quite important for BNCT, the development of lithium target is planned at the 1 st beam line. On the other hand, the stability is more important for research and development of the devices, we plan to use beryllium target which was already established at RIKEN's compact neutron source (RANS).

In this paper, we report the results of calculations for design of the 2 nd beam line.
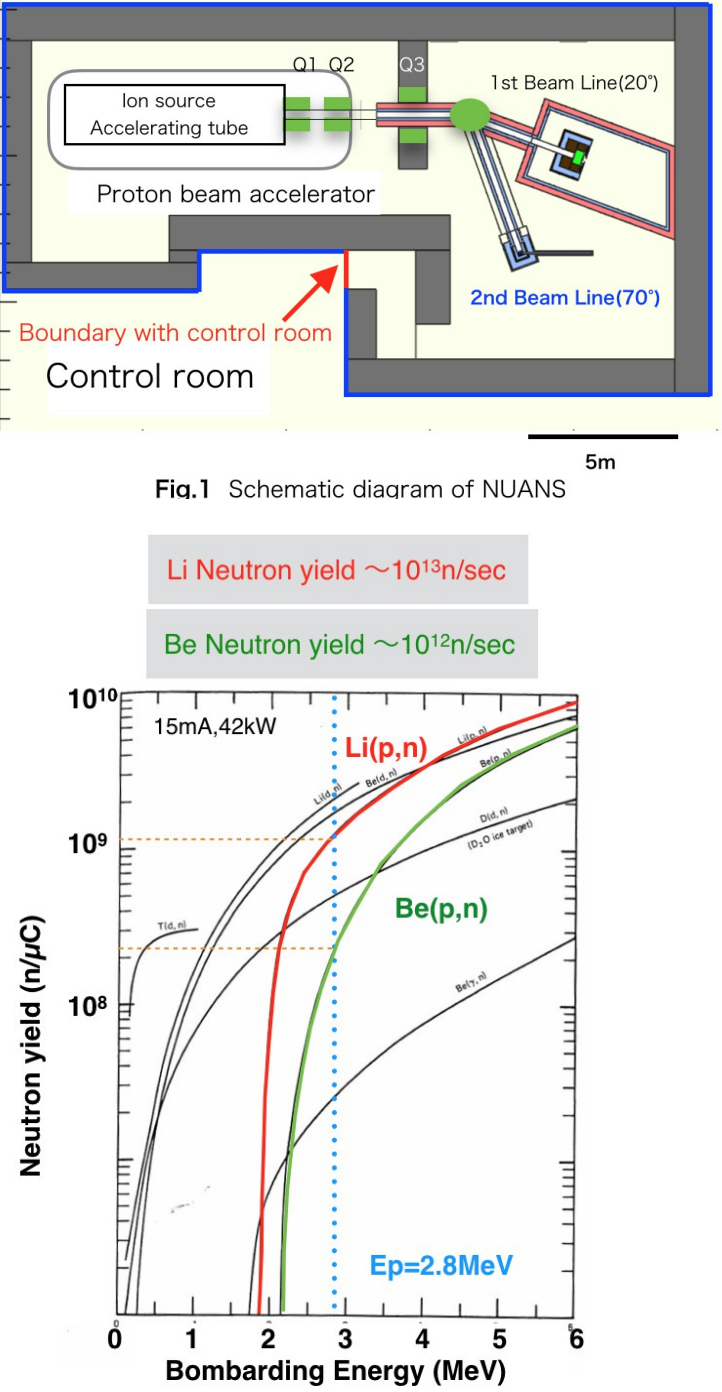

Fig.2 relationship between the energy of the proton beam and the neutron yield of each nuclear reaction. [1]

\section{Calculation for the 2 nd beamline}

The proton beam current used for the $2 \mathrm{nd}$ beam line is $1.5 \mathrm{~mA}$. The size of the neutron generation target with beryllium is $100 \mathrm{~mm}$ in diameter. Polyethylene block is used for a neutron moderator. For radiation safty, the dose at the boundary of the control room should be less than 
$0.1 \mu \mathrm{Sv} / \mathrm{h}$. The shield showld be lighter than 2 tons because of the building structure. The thermal neutron flux of $10^{4} \mathrm{n} \mathrm{cm}^{-2} \mathrm{~s}^{-1}$ is desired for our R\&D. We optimized the design of beam line.

\subsection{Shielding calculation}

We use lead and polyethylene containing $10 \%$ boron oxide for shielding. Polyethylene slows down neutrons to thermal neutrons by elastic scattering with hydrogen. Boron absorbs thermal neutrons and releases gamma rays. Lead shields gamma rays but neutron rays almost penetrate.

Shield calculation was done using PHITS version2.880[2]. We made a sandwich structure of lead and the polyethylene shown in Fig.3. We calculated the the radiation dose and total weight of shielding according to the position of lead (Fig.4). The dose becomes almost flat when the position of lead is farther than $10 \mathrm{~cm}$. We determined the position of $10 \mathrm{~cm}$ to reduce the weight. The weight within the red dotted line was about 1.7 tons. The dose at the boundary of the control room was $0.04 \mu \mathrm{Sv} / \mathrm{h}$.

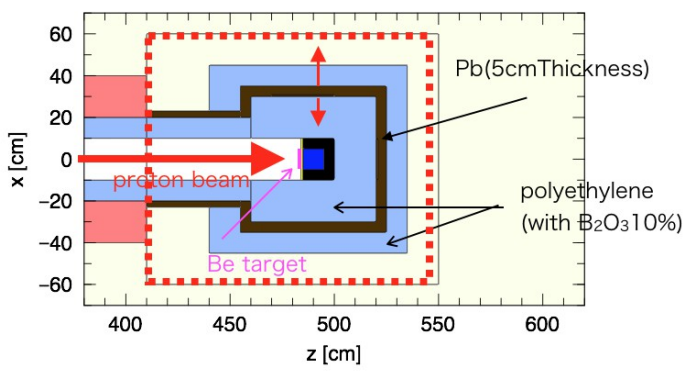

Fig.3 Sandwich structure of 2nd beam line

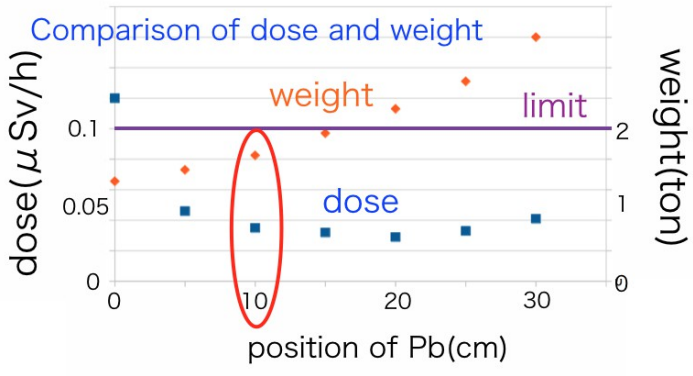

Fig.4 Result of simulation

\subsection{Neutron intensity calculation}

We considered the neutron extraction hole shown in Fig.5. The cross section of the hole was set to $10 \mathrm{~cm} \times 10 \mathrm{~cm}$. We calculated the thermal neutron flux at the downstream of $2 \mathrm{~m}$ from the moderator. Fig.6 shows the flux as a function of the depth of the extraction hole in the moderator. As a result, the thermal neutron flux reached $10^{4} \mathrm{n} \mathrm{cm}^{-2} \mathrm{~s}^{-1}$.

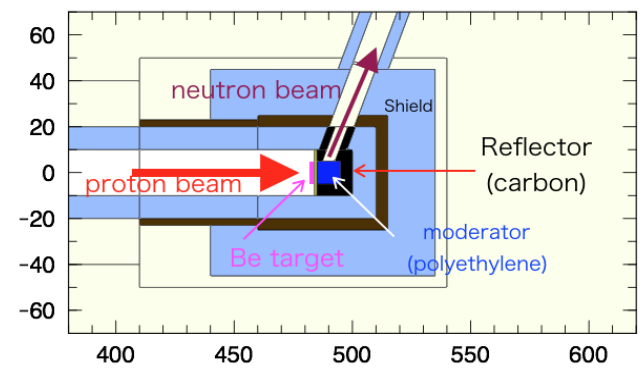

Fig.5 the neutron extraction hole
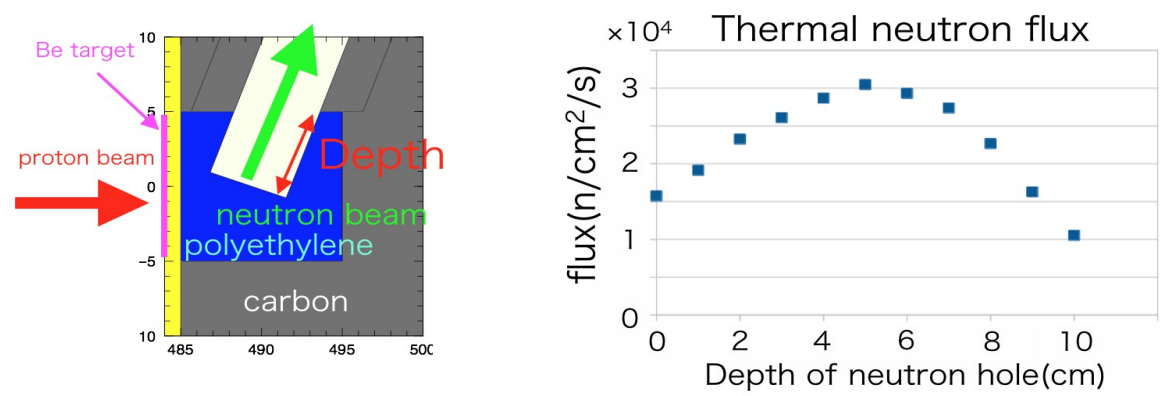

Fig.6 (Left) Hole for neutron extraction

(Right) the flux as a function of the depth

of the extraction hole in the moderator 


\subsection{Calculation of proton beam spreading}

We calculated the spread of the proton beam from the accelerator exit to the neutron generation target. There are three quadrupole magnets to control the proton beam, and a switching magnet to bend the beam (Fig.7). The proton beam must be spread to the area of $80 \mathrm{~mm}$ in diameter on the beryllium target in order to reduce the heat load. We calculated the spreading of the proton beam using TRACE3D[3]. Fig.7 shows the result when the proton beam is most focused on the Be target. The blue and red line shows the horizontal and vertical spread of the proton beam, respectively. We demonstrated the beam transport satisfying the requirement of the heat load.

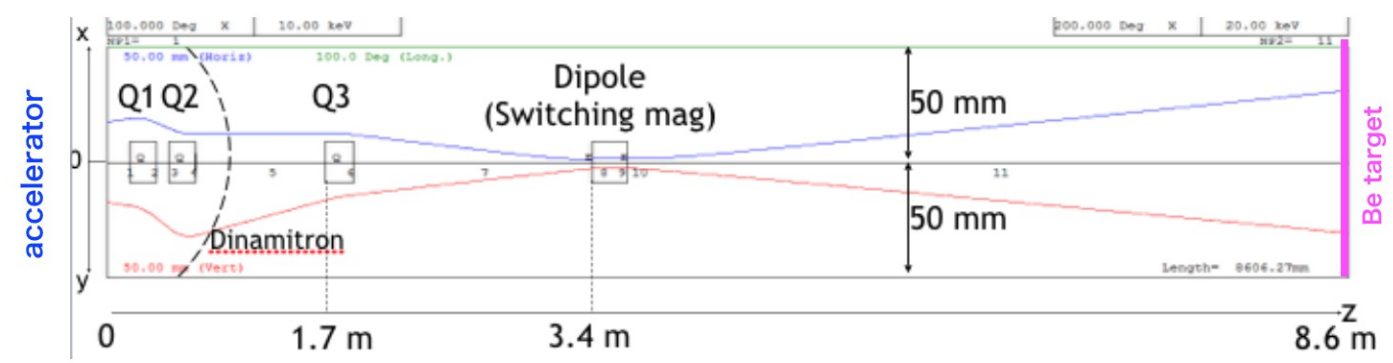

Fig.7 Result of calculation of proton beam spreading

\section{Summary}

We are constradting a compact neutron source at Nagoya University. The simulations for radiation shielding, extraction of neutron beam, and proton beam transport were performed. We demonstrated the feasibility of NUANS 2 nd beam line.

\section{References}

[1] M.R.Hawkesworth, Atomic Energy Review 15 2(1977) P169

[2] T. Sato, K. Niita, N. Matsuda, S. Hashimoto, Y. Iwamoto, S. Noda, T. Ogawa, H. Iwase, H. Nakashima, T. Fukahori, K. Okumura, T. Kai, S. Chiba, T. Furuta and L. Sihver, Particle and Heavy Ion Transport Code System PHITS, Version 2.52, J. Nucl. Sci. Technol. 50:9, 913-923 (2013)

[3] K. R. Crandall, D. P. Rusthoi, ”Trace 3-D Documentation”, LA-UR-97-886 (1997). 